\title{
IMPlementation Of ClOUd SERVices For AdVANCE MANAGEMENT OF STEEL TRANSPORT FOR Continuous Casting Production
}

\author{
Nikita Kudriashov, Ivan Protasov, Sergey Markov, Vyacheslav Potekhin, \\ Yulia Yadgarova, \& Victor Taratukhin
}
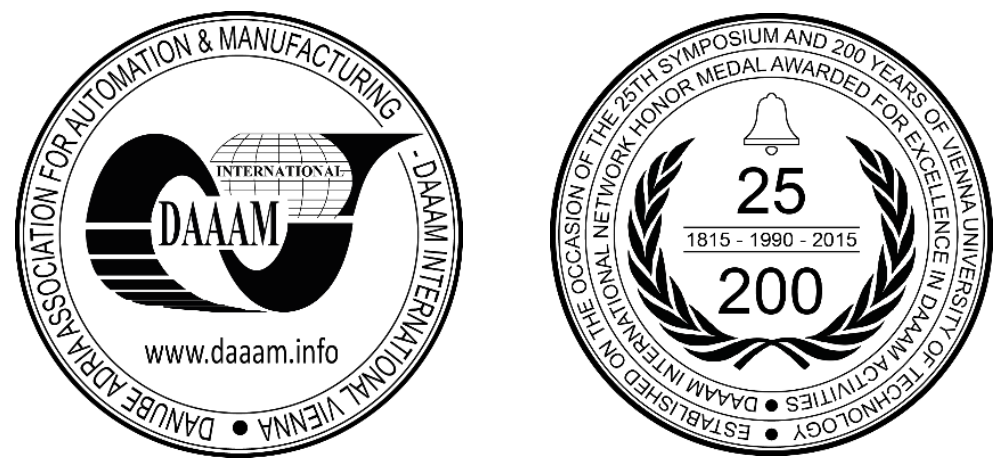

This Publication has to be referred as: Kudriashov, N[ikita]; Protasov, I[van]; Markov, S[ergei]; Potekhin, V[yacheslav]; Yadgarova, Y[ulia] \& Taratukhin, V[ictor] (2016). Implementation of Cloud Services for Advance Management of Steel Transport for Continuous Casting Production, Proceedings of the 27th DAAAM International Symposium, pp.0457-0462, B. Katalinic (Ed.), Published by DAAAM International, ISBN 978-3-902734-08-2, ISSN 1726-9679, Vienna, Austria

DOI: $10.2507 / 27$ th.daaam.proceedings.068

\begin{abstract}
Nowadays, manufacturing is on the threshold of the fourth industrial revolution. It means the increase of enterprise productivity requirements. To improve the productivity, factories have to implement advanced analytical algorithms. It means the increasing demand for big data technology and cloud services for modern manufacturing systems. Therefore, different technologies operating information from Web as well as from field level and top level manufacture information systems should offer high degree of interoperability. For instance, the implementation of Industry 4.0 concepts technologies will be presented on the example of steel transportation for continuous casting production, to access benefits unavailable before.

This paper refers to the problems of Cloud Services and Big Data usage in order to enhance productivity of steel transportation system on metallurgical plant.
\end{abstract}

Keywords: Industry 4.0; Manufacture Execution system; Intelligent control systems; Automation; Cloud services; Casting production

\section{Introduction}

Due to unprecedented development of IT technologies manufactures new opportunities of increasing production efficiency are now possible. Traditional approach to manufacture management is struggling to keep expected outcome. Contemporary realities require production with higher efficiency, quality, and output rates at lower prices in the shortest time possible. Otherwise, there is a high risk of losing orders due to the high-level competition in the market.

Metallurgical industry is one of the most important part of economy [1], therefore it cannot stand apart from the trend which was mentioned above. The question is how can new technologies be implemented for better productivity and resource efficiency for steel transportation system in particular?

Steel transportation for continuous casting production is a system of cranes and rail transporters, which deliver melted steel in ladles for next preparations and casting. A huge machine works following rigid technological process algorithm. 
Involvement to the well-established technological process is unnecessary because of large-scale production, low line flexibility and high level of safety is required. Nevertheless, numerous equipment, which is involved in technological processes, opens great opportunities for maintenance optimisation. Regulations of maintenance, which are able to be more flexible and adaptive, depends on current state on production lines and experience of exploitation in such condition.

To realize the idea of increasing production efficiency by reduction of equipment downtime, we can implement Industry 4.0 concept and IT world technologies[2]. By using Industrial Internet of Things (IIoT) services, we can collect and store data from any devices from production line that provides comprehensive information about production state. The increased data flow can be stored and quickly analysed by Big Data cloud services for statistics management and prognosis of faults. Moreover, implementation of Information Technologies such as machine vision, pattern recognition makes new information about technological process available that was not accessible before. In more detail, about this technologies we will talk further.

\section{Critical overview of works on this field}

First, it is necessary to look at the set of work on this field. As steel mill ladles have relatively short life cycle (on the average, one lining has to be repaired any 50-70 pouring), it is important to analyze its condition. Otherwise, if do not do any necessary repairing procedures, metal breakout will occur. It entails such problems as equipment destructions and, as the result, huge financial losses. The described problem opens a number of possibilities for engineers to make their researches and projects.

As an example, there is a set of works, connected with steel mill ladles lining analysis using IR cameras. All of them has many common features. This approach for ladle monitoring is to place its IR video cameras in robust housings, and locate them in fixed positions where they have a clear view of a filled ladle as it passes by on the gantry crane. Those cameras capture video images of the ladle in real time, and calculate temperatures over the ladle's surface. A few cameras can cover the entire surface of the ladle, but typically, a few relevant areas are predefined for analysis. [3]

Often these solutions differs in the number of IR cameras and hardware configuration. On the Figure 1 the descriptions of three implementations are shown [4]

Another state is that none of this systems store data about previous conditions of ladles. It means that algorithm only analyze the current state of the ladle and can only make the alarm go off, if metal critical overheating has been detected. On the other hand, it shows the Areas on the ladle, where the temperature indicate excessive wear or a defect are highlighted with alarm colours in the on-screen display. This way, the operator gets the location of a potential problem with the refractory [3]. However, we need an experienced operator to analyse the current state of ladle.

Often, this recourse is enough for metal breakout prevention. However, it would be better if we could analyse state of all ladles in any number of pouring. It could implement not only prevention of alarm situation, but plan the usage of current ladle, according to collected data about its specifications.

Big companies work on improving manufacturing processes, considering ideas of Industry 4.0. At Hannover Messe 2014 implementation of smart services of machine maintenance was demonstrated. It was demonstrated in context of the pump unit interfaces connection to Cloud software, which is enabling early prognosis of system errors [6]. In this paper we expand the machine data to cloud connectivity idea for steel transportation system for continuous casting production. It is a complex task to consolidate specific data from IR-cameras and other sensors with cloud services and develop algorithms for reducing amount of unexpected transport system downtimes as well as lowering the maintenance cost. To achieve this aim we have to operate the stored big data quickly which is possible by using cloud technologies.

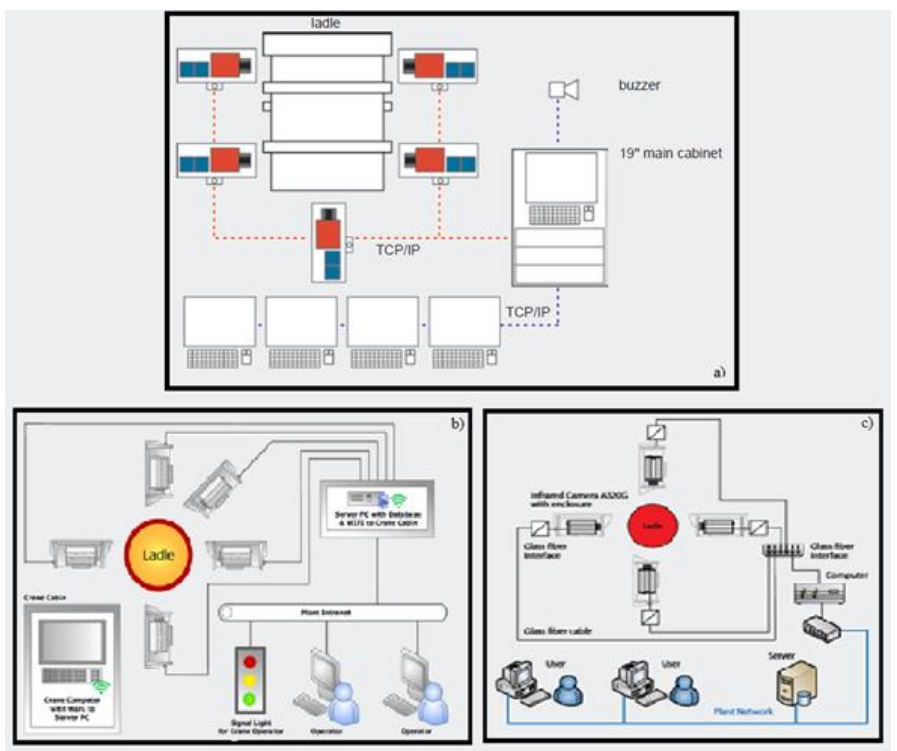

Fig. 1. a - IntraTec [5] b - FLIR solution [3] c - LadlChak [4] 


\section{System description}

Developing system inherits some of the main principals of previous works. However, following issues has to be improved:

- Selection of optimal configuration parameters (number of IR cameras, hardware, connections )

- Software improvement, according to new configuration of the system

- Secondary appointment of the system - multifunctionality, for covering more areas of interest (for example we can reduce speed-measuring sensor by implying machine vision algorithms on IR thermogram).

- Inculcation of Industry 4.0 concepts to implement the system, which can make the decision, based on its experience (Big Data, Neural networks, machine learning algorithms, Cloud services)

Firstly, let us start from the beginning, first question is, how many cameras to chose. There are systems with four cameras (one for each side), and with five cameras (each side and bottom.). To reduce the price of the system, we can reduce one of the cameras, Figure 2.

There are several problems to consider:

- Reducing of one camera entails requirements for its higher resolution. Would it be cheaper?

- Will the quality be good enough to make algorithm work correctly? (On Figure 2 red areas - is the areas of intersection of cameras observation, so the reducing of excess data is needed)

- How accurate would be our system? (Figure 3 - an example of accuracy evaluation for Optris IR Camera )

Therefore, the first step the optimisation task have to be solved. It depends on a huge set of parameters connected with not only camera features, but with specification of current ladle. The optimization goal is to find the cheapest configuration with high value of accuracy and algorithm working time.

Second step of this work is to implement an algorithms, according to chosen configuration of the system. The main requirements to these algorithms:

- Reduce excess information about ladle surface (areas "a" on Figure 2). This goal can be implemented by using such machine vision algorithms as pattern matching, variational methods and contour matching algorithms. In the end, it has to one scan of whole ladle surface.

- Centralized storing of processed ladle surface images. The amount of storing data will expend during the iterations technical process. Big Data technologies, is the only way to solve this problem.

- Decision making algorithms. There are several variants of algorithms for such system. The first one is neural network, which can make a decision, according to its experience,obtained by teaching algorithm. Another way is to use the combination of simple optimization algorithms.

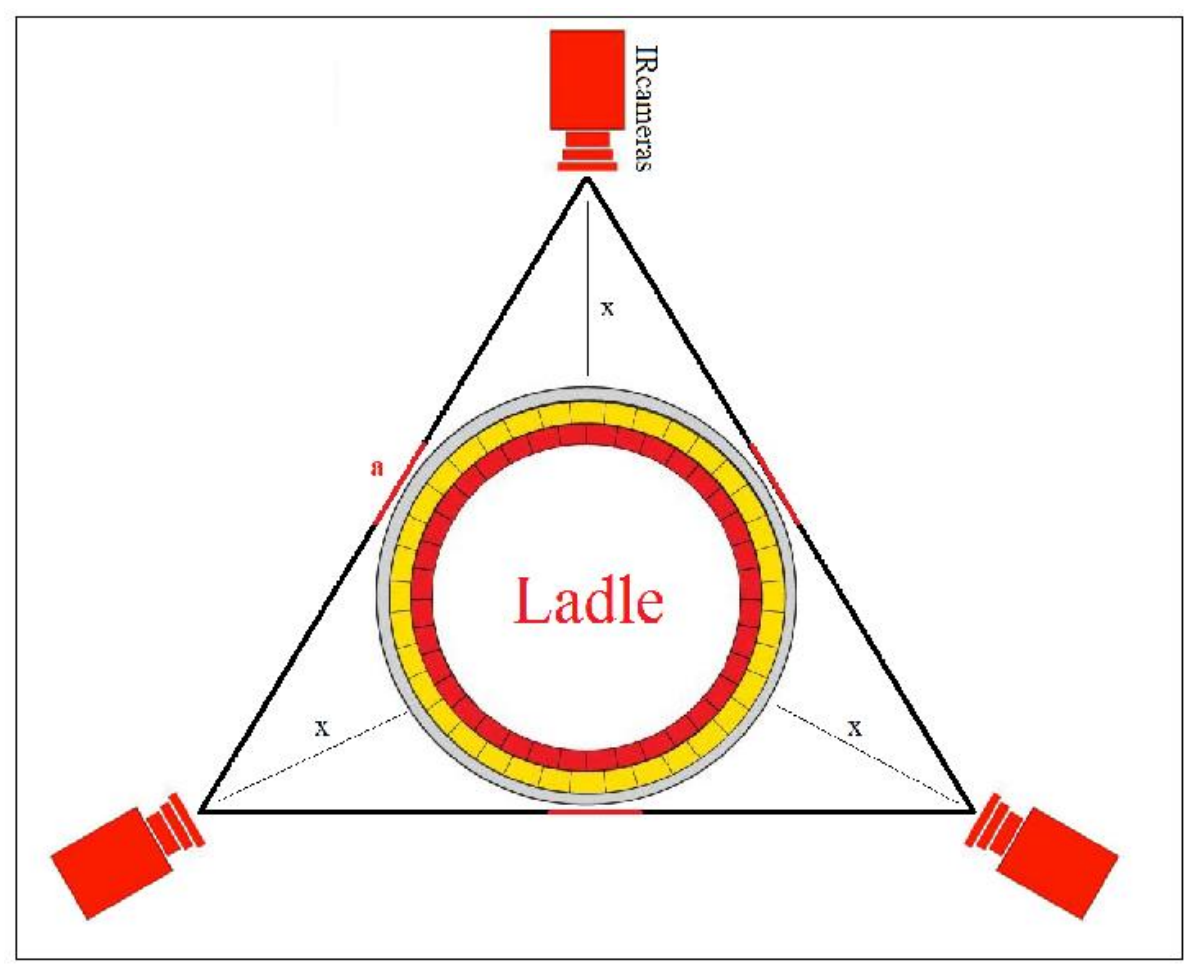

Fig. 2. Three IR camaras configuration 


\section{Optics Calculator}

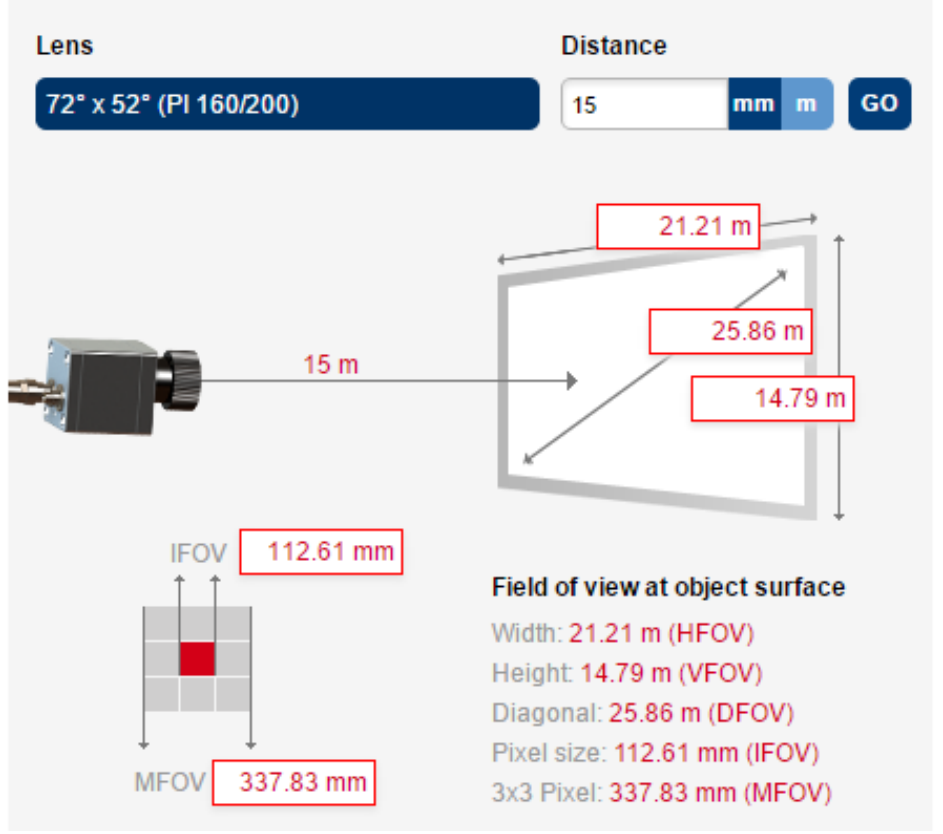

Fig. 3. Parameters evaluation for Optris IR Camera

Thirdly, as it has been said, secondary appointment of the system is multifunctionality. To control steel transport for continuous casting production such parameter as ladle speed has to be measured. Simple contact methods has many limitations. Usage IR Camera can reduce this trivial methods and, by using machine vision algorithms, measure ladle speed according to thermogram[7]. Speed can be measured by using simple tracking algorithms, which takes the most distinctive parts of ladle, and tracks their position during the period of time, then speed values averages, Figure 4.

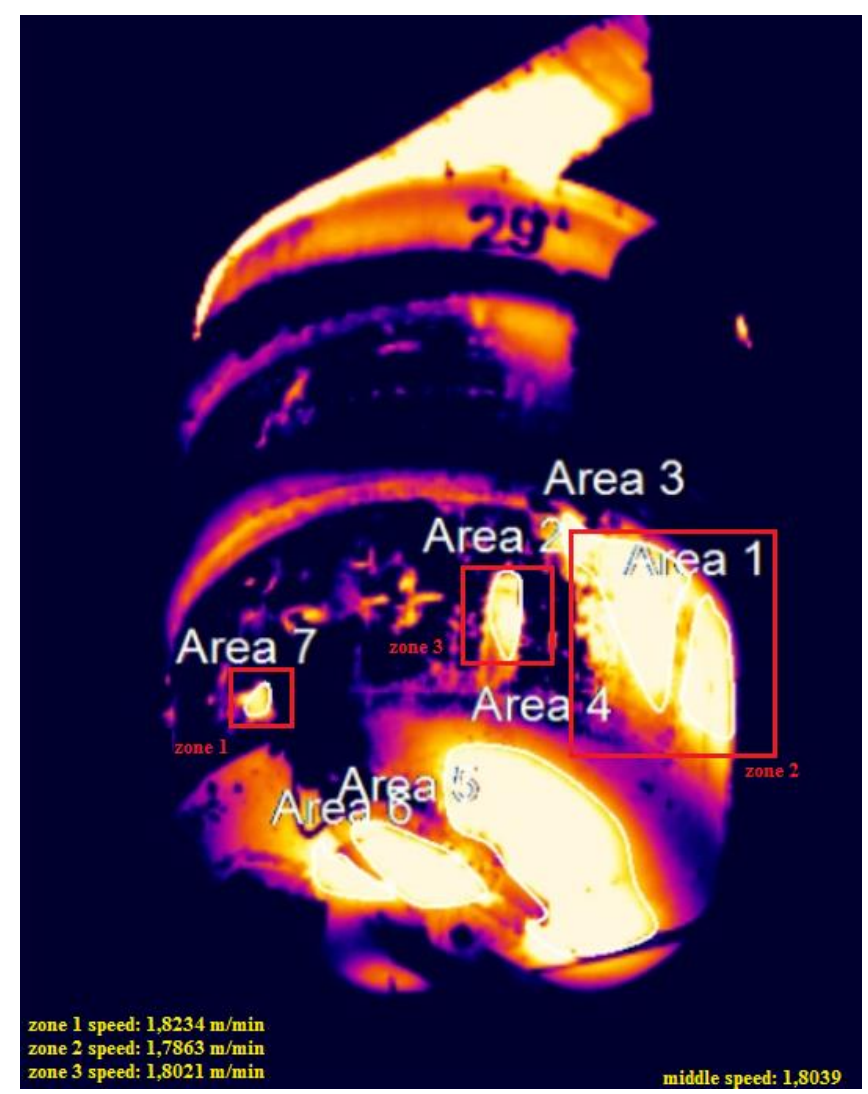

Fig. 4. Tracking of the most distinctive areas of ladle 
Finally, implementation of the IT technologies development achievements can significantly expand functionality of the steel transport control system for continuous casting production. Previous system can only answer the question: is this steel ladle have a latent defect or not? The answer is not provide sufficient information for organizing timely supported maintenance schedule that is extremely important in large-scale production of steel. Resource of the ladles depends on differences parameters, such as construction of the ladle, type of produced steel, technological process, composition of slag and it's properties and many others. If we keep statics of the technological process parameters, we can analyze it and make some prognosis about resource of the ladle. Furthermore, the more statistics data we obtain the more accurate prognosis of the ladle resource we have. Based on this analysis we can plan of maintenance more optimal by ladles resource parameter. Implementation of the proposed idea is reflected in the architecture shown in Figure 5.

The necessary data collected from the IR-cameras analysis system and other shop floor sensors by Manufacture Execution System. Then, MES send the data to the distributed cloud storage. After storing and processing of the received data, the Cloud send to MES optimal plan of equipment maintenance. Using of the cloud storage has the following advantages[8]:

- Availability of relevant information from any plant of metallurgical company,

- Special hardware and software architecture for high performance data operations,

- Using powerful cloud Big Data analytical tools.

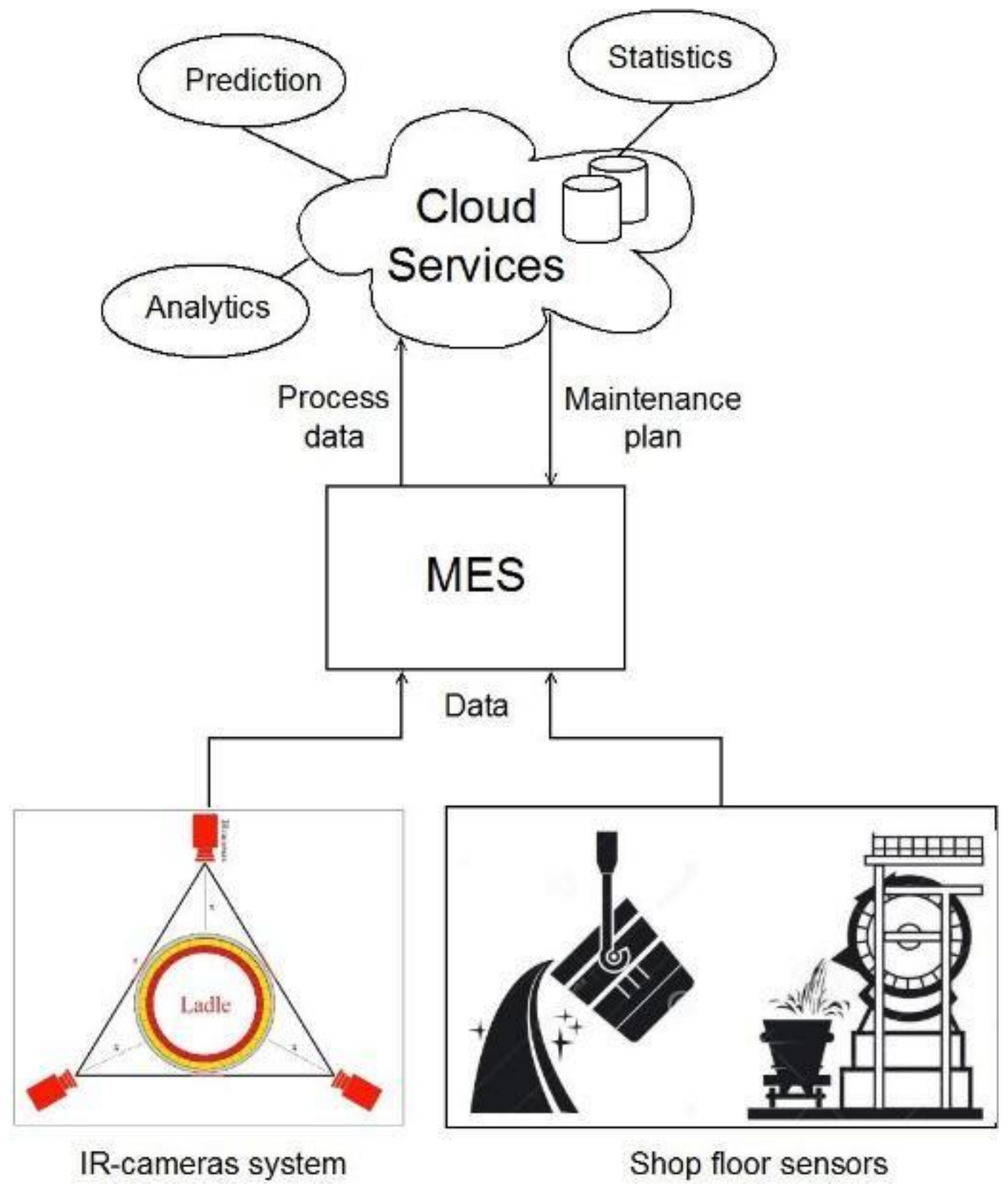

Fig. 5. Architecture of the proposed system 


\section{Conclusion and outlook}

The aim of this work is to propose the implementation of the cloud technologies for steel production costs optimization. According the research, we suggest to reduce cost by optimization of maintenance processes using an example of steel transportation for continuous casting production. As a result we suggest an architecture of equipment state control system which is based on implementation of cloud technologies. Additionally, we consider using of machine vision with IR-cameras for comprehensive analysis of ladle working resource and opening issue of camera quality and quantity, and composition. Furthermore, by using of IR-cameras we can measure ladle speed more precisely by tracking algorithms that will increase functionality of the system. Taking implementation of the system into consideration we have derived main requirements for ladle state recognition algorithm. Through research, we observe the clear trend of convergence of shop floor data and Cloud Services [9]. In this paper we demonstrate how to implement modern trends and technologies in order to improve the efficiency of enterprises.

Beyond the scope of this research there is outstanding issues of IR-cameras control system configuration, implementation of machine vision algorithm, development of analytical algorithms and generation of maintenance schedule, elaboration of data collection. Our future research shall focus on issues mentioned above for further architecture development of factory of future.

\section{References}

[1] http://tpprf.ru/download.php?GET=6LPAY\%2F81BmxbyHP1wg7B0g\%3D\%3D, Chamber of Commerce and Industry of the Russian Federation, Analytical Report of the State of the Steel Iindustry, Accessed on: 2016-08-30

[2] Yerofeyev S.A., Ipatov O.A., Markov S.A., Potekhin V.V., Sulerova A.S. \& Shkodyrev V.P. (2015) Adaptive Intelligent Manufacturing Control System, Proceedings; DAAAM International Symposium of Intelligent Manufacturing and Automation, DAAAM 2015; Vienna; Austria; Volume 26, No. 1, ISSN 2304-1382

[3] http://www.flir.com/uploadedFiles/CS_EMEA/Application_Stories/Media/Downloads/Steel_EN.pdf. Mill Ladle Refractory Monitoring, FLIR solution series, Accessed on: 2016-08-30

[4] LadleCheck. Thermal Imaging System for a Reliable. Condition Monitoring of the Refractory Lining, Automation Technology

[5] Ladle Hot Spot Detection. Thermography-based Ladle Monitoring system. InfraTec.

[6] https://news.sap.com/industry-4-0-two-examples-future-factory/, Industry 4.0: Two Examples for the Factory of the Future, Accessed on: 2016-08-30

[7] Kudriashov N.S., Markov S.A. (2016) Multifunctional diagnostics and process control systems based on Infrared thermography methods and machine vision algorithms, Automated System and Technologies, unpublished.

[8] Gastermann, B., Stopper, M., Kossik, A., Katalinic, B. Secure Implementation of an On-Premises Cloud Storage Service for Small and Medium-sized Enterprises, Proceedings of DAAAM International Symposium on Intelligent Manufacturing and Automation, DAAAM 2014; Vienna; Austria; 26 November 2014 through 29 November 2014, Volume 100, Issue C, 2015, Pages 574-583.

[9] Protasov I.I., Yadgarova Y.V., Potekhin V.V. \& Taratukhin V. (2016) Implementation of Cloud Services for Smart Manufacture, Automated System and Technologies, unpublished 